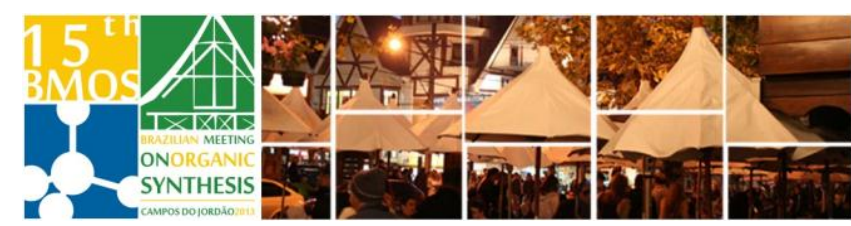

\title{
Design and Synthesis of Diselenides Based-Ionic Liquids
}

\author{
Natasha L. Ferreira*, Vanessa Nascimento and Antonio L. Braga \\ Departamento de Química, Universidade Federal de Santa Catarina (UFSC), Florianópolis/SC, 88040-900 \\ *natasha-If@hotmail.com
}

Keywords:Diselenide, Ionic Liquids, GPX.

\section{INTRODUCTION}

The chemistry involving selenium is essential for maintaining the life of aerobic organisms, in particular because of the protective antioxidant enzyme glutathione peroxidase (GPx), which protects the body from toxic peroxides. Inspired by the GPx enzyme, several classes of new seleniumbased compounds have been prepared for biological studies.

On the other hand, ionic liquids are distinguished by their known catalytic abilities from their high salvation capability, as well as water solubility. Inspired by these properties of ionic liquids and the application range (range of bilogical application of) of biological selenium compounds we planned in this work the synthesis of a new series of ionic liquids containing selenium with new potential biological activitys (Figure 1). Besides, in our strategy, the diselenides designed should contain heteroatom ( $N$ or $\mathrm{O}$ ) in the favorable position to chelate with $\mathrm{Se}$ atom, as well as good solubility in water.

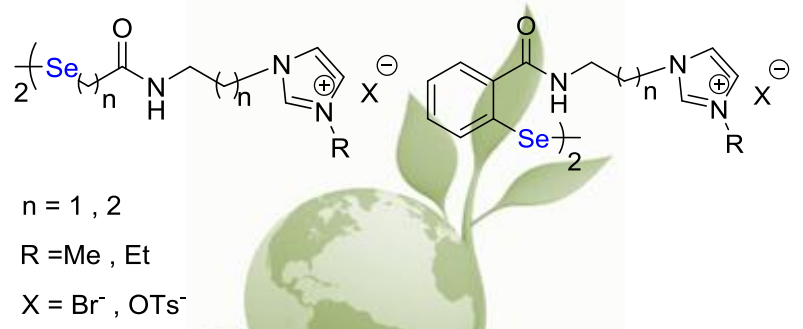

Figure

planned

1.Structures

\section{RESULTS AND DISCUSSION}

The synthesis of the starting materials (Scheme 1) began with the reaction of the corresponding bromoacids with $\mathrm{Na}_{2} \mathrm{Se}_{2}$ generated in situ. ${ }^{3}$

$$
\begin{aligned}
& \mathrm{HO}_{\mathrm{H}} \stackrel{\mathrm{O}}{\mathrm{Br}} \stackrel{\mathrm{NaBH}_{4} / \mathrm{Se}^{0}}{\longrightarrow} \mathrm{HO}_{\mathrm{H}} \stackrel{\mathrm{I}_{\mathrm{n}} \mathrm{Set}_{2}}{\longrightarrow} \\
& \mathbf{1 a} n=1 \quad 2 \mathbf{a}_{n}=1(91 \%) \\
& 1 \mathrm{~b} n=2 \quad 2 b \mathrm{n}=2 \text { (quantitative) }
\end{aligned}
$$

Scheme 1.Synthesis of acid-diselenides 2

With the acid-diselenides in hands, we initially studied the amide bond formation between the aciddiselenide (2b) and 1-(3-aminopropyl) imidazole (3), by testing a series of coupling reagents, such as DCC, EDC, CDI and DIC. EDC (1-Ethyl-3-(3dimethylaminopropyl)-carbodiimide) have showed best better results, giving the target diselenides based-ionic liquid 4 in good yield (96\%) (Scheme 2).

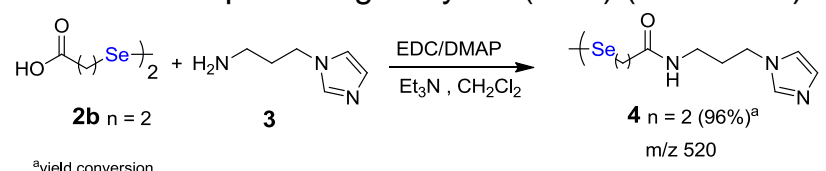

Scheme 2. Synthesis of diselenide based-ionic liquid

To confirm the preparation of the diselenide 4 we show in Figure 2 the mass spectra of its molecular ion $\mathrm{atm} / \mathrm{z} 520$, with the isotopic distribution characteristics of the diselenides.

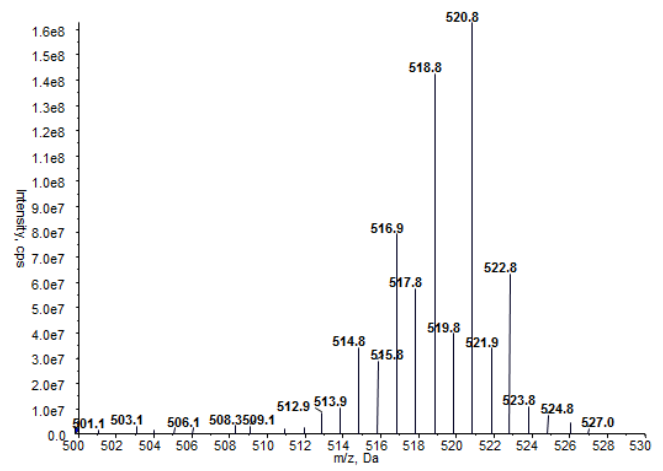

Figure 2. Mass spectrum of the diselenide 4

\section{CONCLUSION}

In summary, we could find a useful route methodology to prepare a structurally important class of selenium compound, the diselenide basedionic liquids. This kind of compound has the potential to mimic the seleno enzyme GPx, since it contains the heteroatom ( $\mathrm{N}$ or $\mathrm{O}$ ) in the favorable position to chelate with $\mathrm{Se}$ atom, that is an important attribute for this activity.

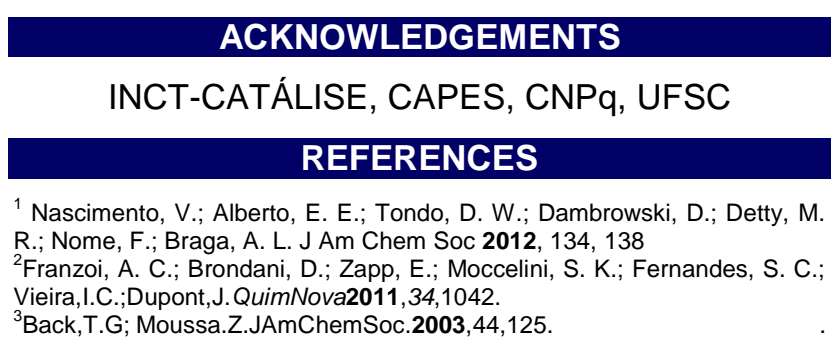

